\title{
Stability analysis of magnetized astrophysical relativistic jets without current sheets
}

\author{
Charalampos Sinnis* and Nektarios Vlahakis \\ Section of Astrophysics, Astronomy and Mechanics, Department of Physics, National and \\ Kapodistrian University of Athens, Panepistimiopolis Zografos, 15784, Athens, Greece \\ E-mail: chsinnisephys.uoa.gr, vlahakisephys.uoa.gr
}

\begin{abstract}
Astrophysical jets are observed as stable structures, extending in lengths several times their radii. The role of various instabilities and how they affect the observed jet properties has not been fully understood. Using the ideal relativistic MHD equations to describe jet dynamics we aim to study the stability properties through linear analysis. In this work we probe stability properties of jets without current sheets and low magnetizations, moving with mildly relativistic Lorentz factors. In particular we find the dispersion relation for kink $(m= \pm 1)$ and pinch $(m=0)$ modes. In the former we find that a wide range of wavelengths $\sim 1-1000$ jet radii equally contributes to the instability with growth rates $\sim$ a few $10^{-3} c / r_{j}$, while in the latter the large wavelengths are more unstable, giving similar growth rates. Evaluating the eigenfunctions of the instability we see that they attain their highest values near the jet boundary, indicating that the instabilities are most likely of a Kelvin-Helmholtz type rather than current driven.
\end{abstract}

High Energy Phenomena in Relativistic Outflows VII - HEPRO VII

9-12 July 2019

Facultat de Física, Universitat de Barcelona, Spain

\footnotetext{
* Speaker.
} 


\section{Introduction}

Plasma jets are widespread in various astrophysical settings, remarkably stable, reaching lengths many times their initial radius. On the other hand, it is well established in laboratory plasma configurations that different kind of instabilities are present and interfere with their structure and dynamics. An important question arises, how the astrophysical counterparts survive and remain intact for such extreme lengths.

An efficient method to study the stability properties of jets is through linear stability analysis. This kind of work have been done for the non-relativistic magnetohydrodynamic regime (see e.g. $[1,2,3,4]$ ) and also for the relativistic regime (see e.g. [5, 6, 7]) where additional effects modify the stability properties.

The assumed unperturbed equilibrium configuration dictates which kind of instability will play the dominant role for the evolution of the jet. So, for low magnetizations kinetic instabilities are more dominant, while for highly magnetized jets current-driven instabilities take over [8]. Also, the kinematic properties are important since by increasing the velocity, either in the form of bulk movement or rotation, in general stabilize jets $[9,10]$.

A model for cylindrical magnetized jets was introduced in [11] where purposefully the magnetic field topology ensure that the outflow has no current sheet at it's boundary surface. This was feasible through the inclusion of thermal pressure int he dynamics of the problem. In the following years there were a series of publications $[12,13,14,15]$ where the stability properties of this configuration were probed via linear analysis. The first two papers were in the non-relativistic regime with [13] adding a shearing attribute to the velocity profile. The latter have relativistic velocities with [15] also exhibiting sheared velocity profiles.

Focusing on studies without sheared velocity profiles $[12,14]$ the main findings were that as the magnetic field strength increases, the stability of current sheet free jets also increases. Additionally, relativistic motion plays a stabilizing role. We conducted a parametric study for the stability properties of current sheet free jets [16] in relation to the Lorentz factor values. We included pinch and kink modes, while our configuration was essentially hydrodynamic, since we focused mainly on the kinetically induced instabilities. A sample of this work is presented in this paper, where we show the stability analysis results for mildly relativistic Lorentz factors and compare with results from [14].

This paper is organized as follows. In section 2 we present the unperturbed jet configuration and the methodology to derive the physical quantities. In section 3 we give a basic outline of the linearization process and the way we treat numerically our perturbed system. In section 4 we present our results and a summary of this work.

\section{Unperturbed jet configuration}

In this section we introduce the jet model used for our stability analysis. This configuration was derived and initially published in [11]. In this section we will briefly present the methodology followed in [11] to build the current sheet free model. Cylindrically symmetric and time independent configuration is assumed, meaning that every physical quantity depends only on radial distance $r$, effectively vanishing any term differentiating with respect to variables $\phi, z$ and $t$, i.e. 
$\partial_{t}=\partial_{\phi}=\partial_{z}=0$. We define jet's velocity profile as $\boldsymbol{v}=v_{z} \hat{z}$, meaning that we have movement along z-axis, with no rotational or radial components. Magnetic fields have $\mathrm{z}$ and $\phi$-component of the form:

$$
\mathbf{B}=B_{\phi}(r) \hat{\phi}+B_{z}(r) \hat{z}
$$

Combination of magnetic field and velocity profiles induce a radial electric field, due to infinite plasma conductivity.

$$
\mathbf{E}=E_{r}(r) \hat{r}=\frac{v_{z}}{c} B_{\phi} \hat{r}
$$

Next step involves momentum equation:

$$
\gamma \rho\left(\frac{\partial}{\partial t}+\boldsymbol{v} \cdot \nabla\right)(\gamma \xi \boldsymbol{v})=-\nabla p+\frac{J^{0} \mathbf{E}+\mathbf{J} \times \mathbf{B}}{c}
$$

where $\gamma$ is Lorentz factor, $\rho$ is rest mass density, $\xi$ is specific enthalpy and $J^{0}\left(=\frac{c}{4 \pi} \nabla \cdot \mathbf{E}\right)$ is the electric charge density. Substituting the physical quantities into eq. 2.3, after the required algebra, we reach it's final Grad-Shafranov equation form:

$$
\frac{r}{2}\left(B_{z}^{2}+B_{\phi}^{2}-E^{2}+8 \pi p\right)^{\prime}+B_{\phi}^{2}-E^{2}=0
$$

Step by step methodology to solve eq. 2.4 is given in [11], we will focus on the provided solutions:

$$
\begin{aligned}
& B_{z}=c a J_{0}(a r)-\frac{2 F}{a^{2}} \\
& B_{\phi}=\frac{1}{\left(1-v_{z}^{2}\right)^{1 / 2}}\left(c a J_{1}(a r)-\frac{F r}{a}\right) \\
& E=\frac{v_{z}}{\left(1-v_{z}^{2}\right)^{1 / 2}}\left(c a J_{1}(a r)-\frac{F r}{a}\right) \\
& p=\frac{1}{4 \pi} F\left(c r J_{1}(a r)-\frac{F r^{2}}{a^{2}}\right)+p_{0}
\end{aligned}
$$

So depending on the choice of the velocity profile the above solutions are regulated accordingly. In order to simplify the model a top hat velocity profile is our choice, meaning that in the jet $\left(r \leq r_{j}\right)$ the velocity is uniform while the environment is static.

\section{Linear stability method}

In this section we will highlight the basic properties of the methodology for studying the linear regime of jet stability. The first step is to linearize the set of equations ruling the dynamics of our perturbed configuration. We assume ideal relativistic MHD and insert small perturbations decomposed into Fourier parts of the form:

$$
\delta Q(r, \phi, z, t)=Q_{1}(r) \exp [i(-\omega t+m \phi+k z)]
$$


where $\omega$ is complex, $m$ integer and $\mathrm{k}$ real. Setting $\omega \in \mathbb{C}$ we follow the temporal approach, meaning that the amplitude of perturbations is time dependent. Using $\omega=\Re \omega+i \mathfrak{I} \omega$ from eq. 3.1 we have:

$$
\delta Q(r, \phi, z, t)=Q_{1}(r) \exp (\mathfrak{I} \omega t) \exp [i(-\Re \omega t+m \phi+k z)]
$$

defining the growth rate of perturbations $\mathfrak{I} \omega$. Whenever $\mathfrak{I} \omega>0$ instability arises, whereas when $\mathfrak{I} \omega=0$ we have marginal stability and when $\mathfrak{I} \omega<0$ a stable state. In this work we only focus on unstable solutions. Our final system after the proper algebra is of the form:

$$
\frac{d}{d r}\left(\begin{array}{l}
y_{1} \\
y_{2}
\end{array}\right)+\frac{1}{\mathscr{D}}\left(\begin{array}{ll}
\mathscr{F}_{11} & \mathscr{F}_{12} \\
\mathscr{F}_{21} & \mathscr{F}_{22}
\end{array}\right)\left(\begin{array}{l}
y_{1} \\
y_{2}
\end{array}\right)=0
$$

where $y_{1}=i \frac{r V_{1 r}}{\omega_{0}}, y_{2}=\Pi_{1}+\frac{y_{1}}{r} \frac{d \Pi_{0}}{d r}$ and $\omega_{0}=\omega-k V_{z}-m V_{\phi} / r$. Coefficients $\mathscr{F}$ and $\mathscr{D}$ are determinants, functions of the unperturbed physical quantities and their derivatives. The unknowns are $y_{1}$ and $y_{2} . y_{1}$ is related to the radial component of the Lagrangian displacement and $y_{2}$ to the variation of the total pressure.

The solution for jet's environment can be found analytically, due to the assumptions made for this regime. The ambient medium is static and unmagnetized, with constant density and thermal pressure profiles. System 3.3 simplifies to a Bessel differential equation and the solution is a first type Hankel function of first kind $\left(H_{m}^{(1)}(r)=J_{m}(r)+i Y_{m}(r)\right)$, satisfying the Sommerfeld radiation condition.

For the jet's interior the solution can be found numerically, by integrating from the axis (paying attention to the regularity conditions at $r=0$ ) to the jet boundary $r=r_{j}$. At this point we apply boundary conditions to match the $r<r_{j}$ area solutions to the ambient medium. We require at $r=r_{j}$ both $y_{1}$ and $y_{2}$ to be continuous.

\section{Results}

The set of parameters in our integrations are the same used in one of the cases in [12], i.e. $\eta=\frac{\rho_{j}}{\rho_{e}}=0.1, M=4$ and $\beta=1 / 4$, where $\rho_{j}$ is the density of jet on axis, $\rho_{e}$ is ambient medium density, $M$ and $\beta$ are the values of Mach number and plasma beta respectively on the axis $r=0$. From these three quantities and the equation of state $\left(p / \rho^{\Gamma}=\right.$ constant, where $\Gamma=5 / 3$ is the polytropic index) we can find the values of $p_{0}$ and the distribution of $\rho$ with $r$. Once we find the distribution of rest density and pressure, we vary the bulk speed of the jet, studying the stability properties of current sheet free jets for various Lorentz factors. However, contrary to [14] we do not change the distribution of the rest density (and the magnetization) in the jet, focusing only on how the Lorentz factor affects the stability properties.

So, in this paper we will show the dispersion relations, $\omega=\omega(k)$, for mildly relativistic Lorentz factors $\gamma=2$ and $\gamma=5$. The azimuthal wavenumber $m$ takes values 0 and \pm 1 for pinch and kink modes respectively.

\section{$4.1 \gamma=2$ case}

We first show results for the $\gamma=2$ case in fig. 1. It should be noted that in our figures $k$ range is not the same for every plot. We omit to show higher values of $\mathrm{k}$ when the curves just follow 

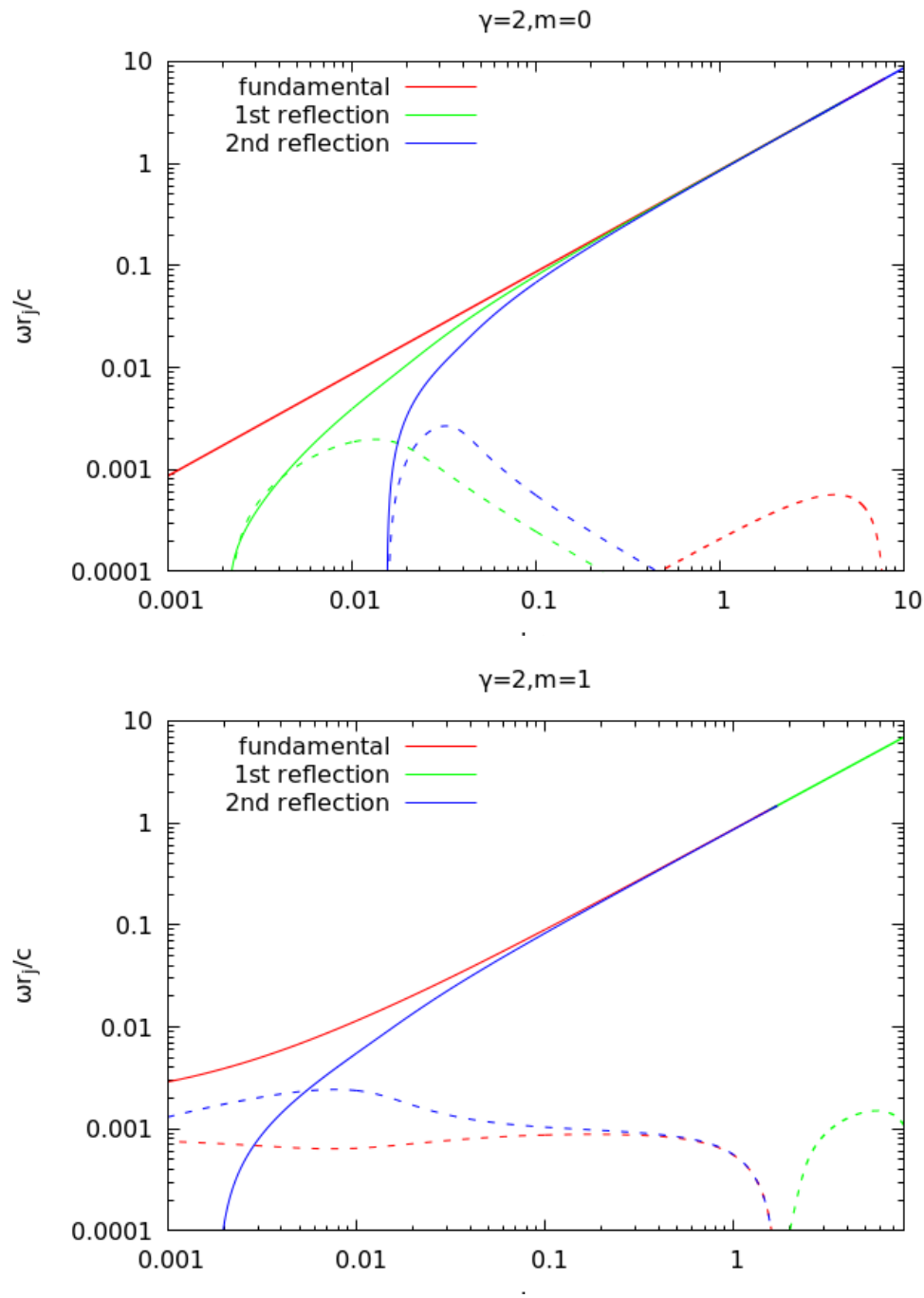

$\gamma=2, m=-1$

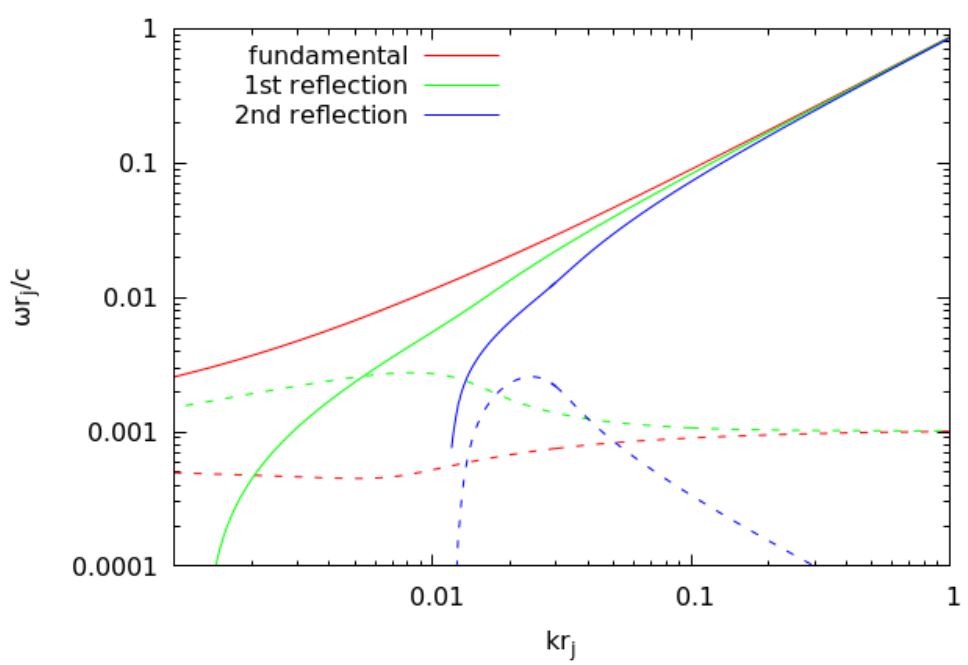

Figure 1: Dispersion plot for $\gamma=2$ and $m=0$ (top), $m=1$ (middle), and $m=-1$ (bottom). Solid and dashed lines are $\mathfrak{R} \omega$ and $\mathfrak{I} \omega$ parts respectively. Unit of $\omega$ is jet's radius light crossing time. There are three oscillatory modes, fundamental in red, first and second reflection modes in green and blue, respectively. 

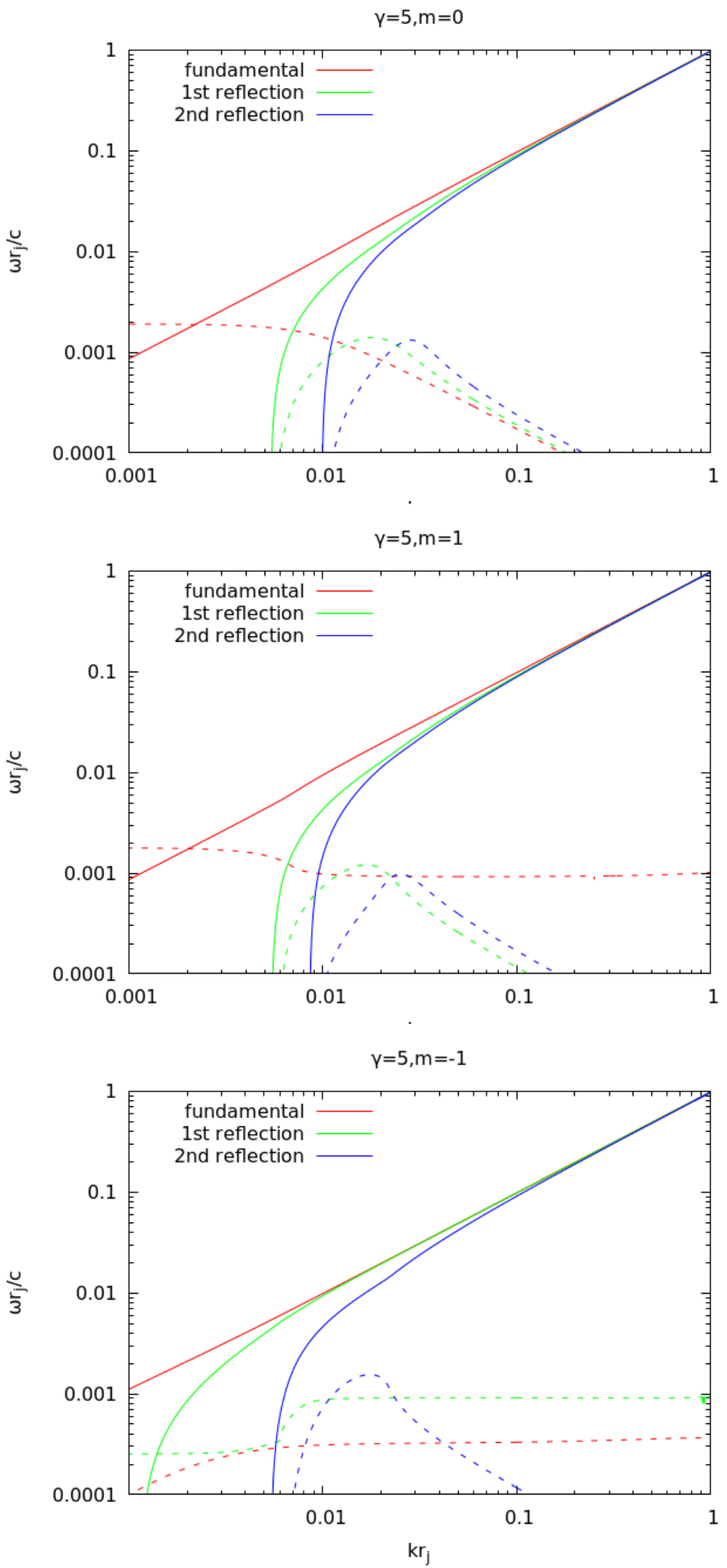

Figure 2: Similar to fig. 1 but for $\gamma=5$. 
the trend set by $k r_{j}<1$ and do not show different behavior for high $k$ values. As we can see, for $m=0$ the fundamental mode is more unstable at small wavelengths while the first and the second reflection modes are important over large wavelengths. Also, the maximum growth rate $\mathfrak{J} \omega$ of the fundamental mode is smaller compared to the reflection modes' respective values. The overall maximum $\mathfrak{I} \omega$ is approximately $3 \times 10^{-3} c / r_{j}$ at $k r_{j} \simeq 0.03$.

For $m=1$ we have instabilities equally important over the entire $k$ range. First reflection mode is present for $k \gtrsim 1$ while fundamental and second modes are unstable for $k \in[0.001,1]$ terminating for $k \sim 1$. The maximum value for $\mathfrak{I} \omega$ is $\sim 2.5 \times 10^{-3} c / r_{j}$ at $k \sim 0.01$. For $m=-1$, again the maximum $\mathfrak{I} \omega$ resides at $k r_{j} \sim 0.01$ and has a value $\simeq 3 \times 10^{-3} c / r_{j}$. A significant difference however is that the fundamental and first mode are equally important over the complete $k$ range and converge towards the same $\mathfrak{I} \omega$ value for large $k r_{j}>0.1$.

Comparing to [14] our configuration is slightly less stable, by a factor of 1.5 , essentially sharing the same perturbation growth timescales. This statement does not hold as magnetization increases, as growth rates lessen significantly in [14] leading to the conclusion that increasing magnetization stabilize the current sheet free configurations.

\section{$4.2 \gamma=5$ case}

Moving to the $\gamma=5$ case, we see in fig. 2 that the maximum growth rate is $\mathfrak{I} \omega \sim 2 \times 10^{-3} c / r_{j}$ in all cases, meaning that both pinch and kink modes need the same time to develop and equally affect the unperturbed configuration. Our solutions are more unstable compared to [14], most likely because they are less magnetized, agreeing with the conclusion stated at the end of sec. 4.1.

For $m=0$ we can see that the unstable region includes only big wavelengths, $k r_{j} \lesssim 3 \times 10^{-2}$, whereas kink mode plots have their unstable region the entire $k$ range. This is a trend holding from sec. 4.1 where higher $k$ have lower $\mathfrak{I} \omega$ components than their low $k$ counterparts, meaning that for pinch mode we expect bigger wavelengths to be dominant. Reflection modes in every $\gamma=5$ figures are centered approximately around $k r_{j} \simeq 2 \times 10^{-2}$, except for 1 st reflection mode in fig. 2 bottom panel which spans over whole $k$ range. In general, reflection modes behave similarly and become effectively unstable for a certain $k$ interval around the value mentioned above.

Fig. 3 shows the eigenfunctions for $\gamma=5, m=1, k r_{j}=1.77, \Re \omega r_{j} / c=1.082 \times 10^{-2}$ and $\mathfrak{I} \omega=$ $1.2 \times 10^{-3} r_{j} / c$. Clearly the perturbations attain their maximums and "oscillate" towards the jet boundary, indicating that the main instability is kinematic, most likely of Kelvin-Helmholtz type caused by the velocity difference between the unmagnetized environment and jet.

\section{Conclusions}

In this work we studied jets without currents sheets at their boundary surface, through linear stability analysis. We presented dispersion relation curves and their parametric dependence on two relativistic Lorentz factors $\gamma=2, \gamma=5$ and three azimuthal wavenumbers $m=0, \pm 1$. For pinch modes we saw that small wavenumbers host solutions with larger $\mathfrak{I} \omega$ values in comparison with the large wavenumber regions, meaning that they are less stable. Kink modes behave quite similarly for both Lorentz factors, having solutions covering the entire range of $k$. Their $\mathfrak{I} \omega$ maxima are located near $k r_{j} \sim 0.01$. In general maximum $\mathfrak{I} \omega$ values are not affected essentially by the different Lorentz factors in the mildly relativistic regime and have an approximate value of $2 \times 10^{-3} \mathrm{c} / \mathrm{r}_{j}$. 
$\gamma=5, m=1$
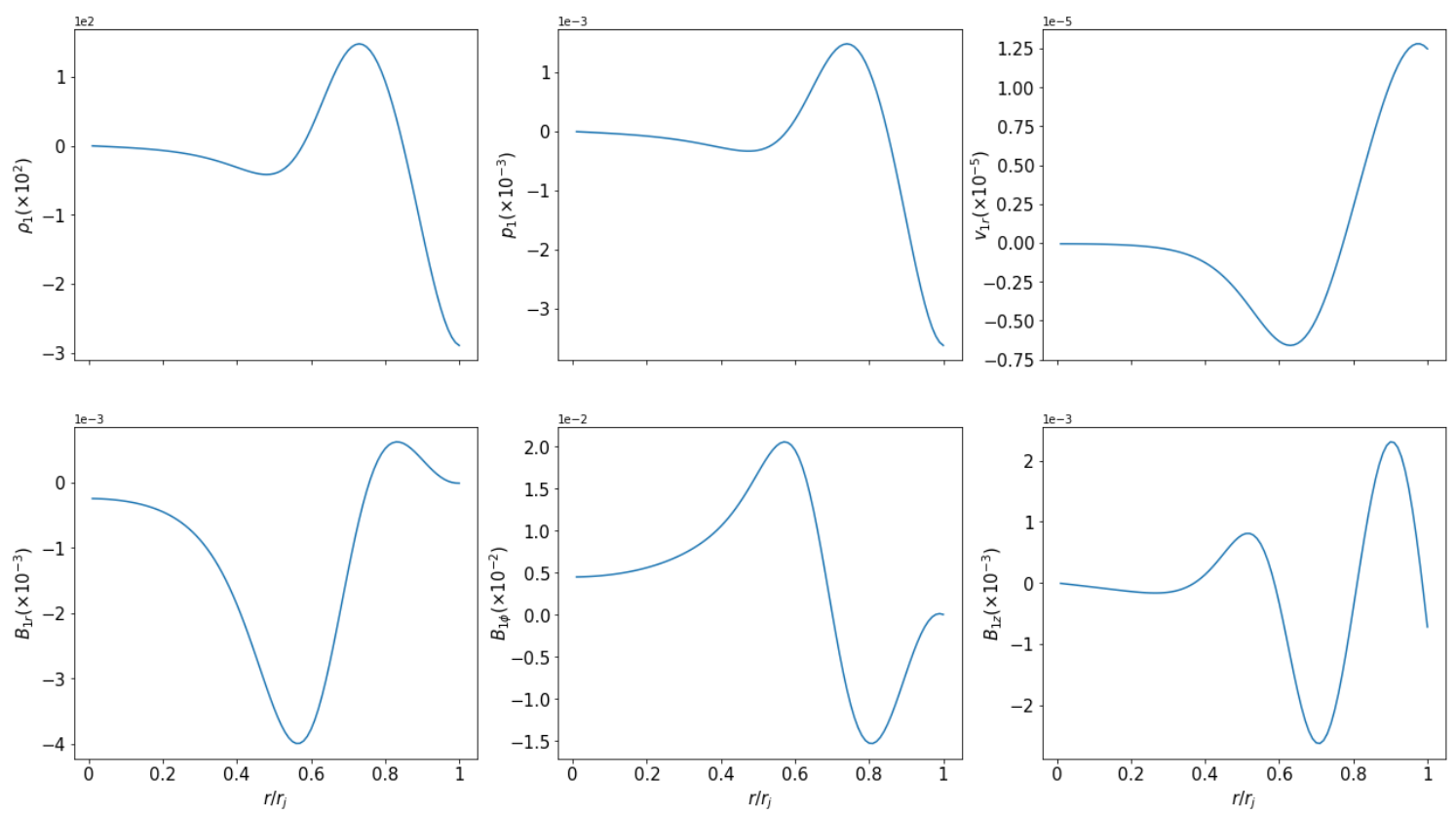

Figure 3: Eigenfunctions plotted versus $r$. The real components of the perturbations are drawn in the plots above. Beginning from top left corner and moving clockwise we have perturbation of density, thermal pressure, radial component of velocity, $\mathrm{z}, \phi$ and radial component of magnetic field.

Our results are in good agreement with the unmagnetized case of [14], quite reasonable as our configuration remains hydrodynamical, even though our plasma $\beta$ is small. Comparing to their magnetized cases our jet is more unstable, a result supporting the fact that current sheet free jets' stability increases as magnetization values increases. Finally, we plotted the eigenfuctions provided by our solution for a specific set of parameters $(\gamma, k, m, \Re \omega, \mathfrak{I} \omega)$. Common trend for every perturbed quantity is that their maxima are located near the jet boundary, enforcing the idea that the main instability contributing to the disturbance of the outflow is the Kelvin-Helmholtz.

\section{Acknowledgements}

This research is co-financed by Greece and the European Union (European Social Fund- ESF) through the Operational Programme "Human Resources Development, Education and Lifelong Learning" in the context of the project "Strengthening Human Resources Research Potential via Doctorate Research" (MIS-5000432), implemented by the State Scholarships Foundation (IKY).

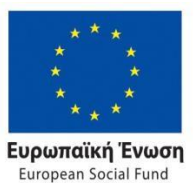

Operational Programme Human Resources Development, Education and Lifelong Learning

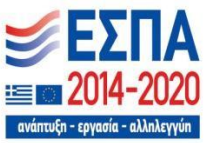

Co-financed by Greece and the European Union 


\section{References}

[1] G. Bodo, S. Massaglia, A. Ferrari and E. Trussoni, Kelvin-Helmholtz instability of hydrodynamic supersonic jets., A\&A 283 (1994) 655.

[2] S. Appl, T. Lery and H. Baty, Current-driven instabilities in astrophysical jets. Linear analysis, A\&A 355 (2000) 818.

[3] H. Cohn, The stability of a magnetically confined radio jet, ApJ 269 (1983) 500.

[4] E. Sobacchi and Y. E. Lyubarsky, External confinement and surface modes in magnetized force-free jets, MNRAS 473 (2018) 2813.

[5] P. E. Hardee, Stability Properties of Strongly Magnetized Spine-Sheath Relativistic Jets, ApJ 664 (2007) 26 [0704.1621].

[6] Y. N. Istomin and V. I. Pariev, Stability of a relativistic rotating electron-positron jet: non-axisymmetric perturbations, MNRAS 281 (1996) 1 [astro-ph/9512145].

[7] R. Narayan, J. Li and A. Tchekhovskoy, Stability of Relativistic Force-free Jets, ApJ 697 (2009) 1681 [0901.4775].

[8] G. Bodo, G. Mamatsashvili, P. Rossi and A. Mignone, Linear stability analysis of magnetized relativistic jets: the non-rotating case, MNRAS 434 (2013) 3030 [1307. 6388].

[9] E. Sobacchi, Y. E. Lyubarsky and M. C. Sormani, Kink instability of force-free jets: a parameter space study, MNRAS 468 (2017) 4635 [1702.03151].

[10] G. Bodo, G. Mamatsashvili, P. Rossi and A. Mignone, Linear stability analysis of magnetized relativistic rotating jets, MNRAS 485 (2019) 2909 [1902 . 10781 ].

[11] K. N. Gourgouliatos, C. Fendt, E. Clausen-Brown and M. Lyutikov, Magnetic field structure of relativistic jets without current sheets, MNRAS 419 (2012) 3048 [1110.0838].

[12] J. Kim, D. S. Balsara, M. Lyutikov, S. S. Komissarov, D. George and P. K. Siddireddy, On the linear stability of magnetized jets without current sheets - non-relativistic case, MNRAS 450 (2015) 982 [1411.2504].

[13] J. Kim, D. S. Balsara, M. Lyutikov and S. S. Komissarov, On the linear stability of sheared and magnetized jets without current sheets - non-relativistic case, MNRAS 461 (2016) 728 [1603.00341].

[14] J. Kim, D. S. Balsara, M. Lyutikov and S. S. Komissarov, On the linear stability of magnetized jets without current sheets - relativistic case, MNRAS 467 (2017) 4647 [1702 . 03882].

[15] J. Kim, D. S. Balsara, M. Lyutikov and S. S. Komissarov, On the linear stability of sheared and magnetized jets without current sheets - relativistic case, MNRAS 474 (2018) 3954.

[16] C. Sinnis, Stability analysis of astrophysical magnetized jets without current sheets, MSc Thesis, National and Kapodistrian University of Athens, 2016. 